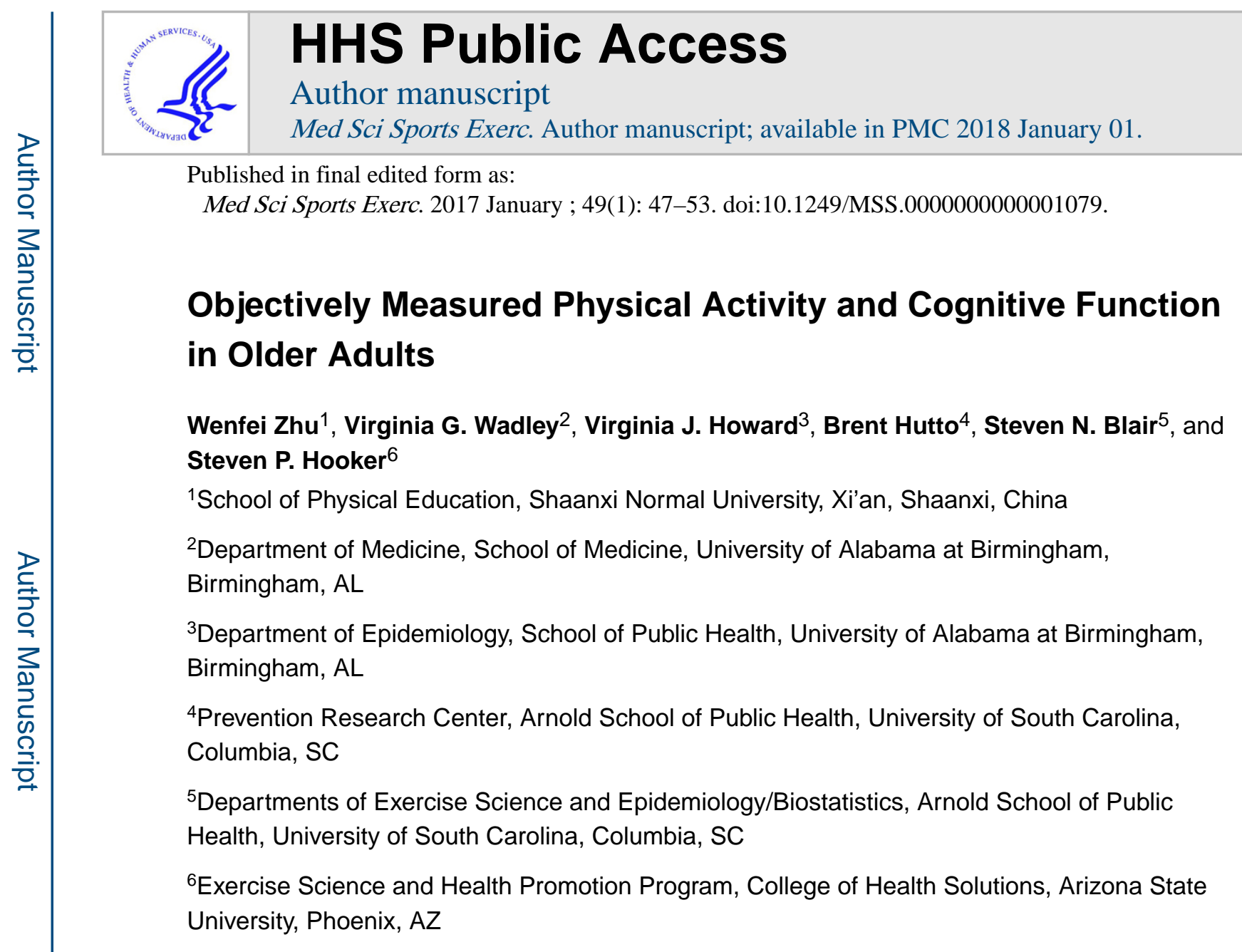

Published in final edited form as:

Med Sci Sports Exerc. 2017 January ; 49(1): 47-53. doi:10.1249/MSS.0000000000001079.

\title{
Objectively Measured Physical Activity and Cognitive Function in Older Adults
}

\author{
Wenfei Zhu ${ }^{1}$, Virginia G. Wadley ${ }^{2}$, Virginia J. Howard ${ }^{3}$, Brent Hutto ${ }^{4}$, Steven N. Blair ${ }^{5}$, and \\ Steven P. Hooker \\ ${ }^{3}$ Department of Epidemiology, School of Public Health, University of Alabama at Birmingham, \\ ${ }^{4}$ Prevention Research Center, Arnold School of Public Health, University of South Carolina, \\ ${ }^{5}$ Departments of Exercise Science and Epidemiology/Biostatistics, Arnold School of Public \\ University, Phoenix, AZ
}

\begin{abstract}
Purpose-Emerging evidence suggests physical activity (PA) is associated with cognitive function. To overcome limitations of self-report PA measures, this study investigated the association of accelerometer-measured PA with incident cognitive impairment and longitudinal cognition among older adults.
\end{abstract}

\begin{abstract}
Methods-Participants were recruited from the cohort study, REasons for Geographic and Racial Differences in Stroke (REGARDS), in U.S.. Accelerometers provided PA measures, including percent of total accelerometer wearing time spent in moderate-to-vigorous intensity PA (MVPA $\%$ ), light-intensity PA and sedentary time, for 4-7 consecutive days at baseline. Cognitive impairment was defined by Six-Item Screener. Letter fluency, animal fluency, word list learning and Montreal Cognitive Assessment (orientation and recall) were conducted to assess executive function and memory.
\end{abstract}

\footnotetext{
Corresponding Author: Wenfei Zhu, Ph.D., School of Physical Education, Shaanxi Normal University, Xi'an, Shaanxi, P. R. China; No. 620, West Chang'an Avenue, Chang'an District, Xi'an 710119, Phone: 86-17792321530; Fax: NA, wzhu @ snnu.edu.cn. Conflicts of Interest and Source of Funding: None. The results of the present study do not constitute endorsement by ACSM. The results of the study are presented clearly, honestly, and without fabrication, falsification, or inappropriate data manipulation.

Publisher's Disclaimer: Medicine \& Science in Sports \& Exercise ${ }_{\circledR}$ Published ahead of Print contains articles in unedited manuscript form that have been peer reviewed and accepted for publication. This manuscript will undergo copyediting, page composition, and review of the resulting proof before it is published in its final form. Please note that during the production process errors may be discovered that could affect the content.
} 
Results-Participants ( $\mathrm{N}=6,452,69.7 \pm 8.5 \mathrm{yr}, 55.3 \%$ women, $30.5 \%$ black) with usable accelerometer and cognition measures spent extremely limited time in MVPA $(1.5 \% \pm 1.9 \%$ of accelerometer wearing time). During an average of 3 years of follow-up, 346 cases of incident cognitive impairment were observed. After adjustments, participants in higher MVPA\% quartiles had a lower risk of cognitive impairment (i.e. Quartile 2: OR=0.64, 95\% CI: 0.48-0.84), and better maintenance in executive function ( $\searrow 0.03 \mathrm{z}$-score units) and memory ( $\searrow 0.12 \mathrm{z}$-score units), compared with Quartile $1(\mathrm{P}<0.05)$. Stratified analyses showed the same association among white adults, but higher MVPA\% was associated with better maintenance of only memory among black adults. No significance was found for light-intensity PA or sedentary time.

Conclusion-There was a dose-response relationship between MVPA\% and cognitive function in older adults, with higher levels associated with a $36 \%$ lower risk of cognitive impairment and better maintenance of memory and executive function over time, particularly in white adults.

\section{Keywords}

cogntive decline; memory; executive function; accelerometer; aging population

\section{Introduction}

As the population ages, cognitive impairment, including Alzheimer's disease and other forms of dementia as well as lesser declines associated with aging, has become one of the greatest health threats. The total number of new cases of dementia each year worldwide is nearly 7.7 million, and the total number worldwide is projected to nearly double every 20 years, to 65.7 million in 2030 and 115.4 million in 2050 (36).

Engagement in physical activity (PA) can positively influence the health of older adults and reduce the risk of many chronic diseases (28). Previous studies support the assertion that PA delays the onset of cognitive decline and the incidence of dementia or Alzheimer's disease associated with aging $(1,2,31)$. Most existing studies, however, have relied on self-reported PA assessments, which may be influenced by recall biases, health status, depression, cognitive ability and other factors, especially for older adults. Objective PA measures have been increasingly used to overcome limitations of self-report measures, but the sample sizes of those present studies were relatively small $(10,32)$. More objective assessments of PA are needed to better define the dose-response association of various PA outcomes with cognitive function.

Additionally, important differences exist in PA levels across racial/ethnic groups (29). Racial/ethnic minorities are more likely to be misclassified by many self-report questions (4). To date, few investigations are based on national studies including a large proportion of black participants. A better understanding of the relationship between objectively measured PA and cognition in different racial/ethnic groups would significantly add to our knowledge of how to best promote healthy cognitive aging. Thus, the purpose of this study was to investigate the association of objectively measured PA with longitudinal cognitive function (including global cognitive function, memory and executive function) in white and black older adults. 


\section{Methods \\ Study Cohort}

The Reasons for Geographic and Racial Differences in Stroke (REGARDS) study is a national longitudinal study of black and white older adults, enrolled January 2003-October 2007 in United States (19). REGARDS is designed to investigate causes of regional and racial disparities in stroke mortality. Using a computer-assisted telephone interview, trained interviewers obtained demographic information and medical history. In-home brief physical examination was conducted 3-4 weeks after the telephone interview. Participants are followed every 6 months for possible stroke outcomes, annually for global cognitive status, and every two years for assessment of memory and executive function. An ancillary accelerometer study was approved in October 2008 for implementation into the ongoing study (20). A question was added during telephone follow-up asking participants if they were willing to wear an accelerometer and complete a daily activity log for one week. Upon acceptance, an accelerometer and log sheet were mailed to participants with instructions. Detailed design and methods have been described elsewhere (20). All participants gave informed consent, and the study was approved by the institutional review boards of Arizona State University, University of South Carolina, and University of Alabama at Birmingham.

Participant characteristics were collected at time of enrollment into the parent REGARDS study. Age, race, sex, region of current residence (southeastern U.S. Stroke Belt vs. rest of the U.S.), highest education level, smoking (current, never, past), physician diagnosis of stroke, history of hypertension, and health status were defined by self-report. Body mass index (BMI) was determined from measured height and weight. Blood pressure was defined as the average of two measurements taken using a standard protocol. Diabetes was defined as fasting glucose level $>126 \mathrm{ml} / \mathrm{dL}$ (or $>200 \mathrm{ml} / \mathrm{dL}$ if participant was non-fasting), or selfreported medication use for glucose control. Of the 12,146 participants who consented to wear an accelerometer, 8,096 provided data after exclusions for failure to wear or return the device, device error, missing log sheet, and noncompliance with the criterion of $>4$ days with $>10 \mathrm{hr} /$ day of wear time. Details of inclusion and exclusion are described elsewhere $(18,20)$.

Cognitive impairment was defined by each participant's score on the Six Item Screener (SIS) (9). SIS scores range from 0 to 6 , with a score of $<4$ correct responses indicating cognitive impairment (9). No participant in this cohort developed incident cognitive impairment before PA variables were measured. After sensitivity analysis, cognitive tests conducted \pm 12 months of PA measurement were selected to achieve the largest sample size without impacting the results significantly. Participants were excluded if they: 1 . had no record of SIS within \pm 12 months of PA assessment ( $n=696) ; 2$. were identified as cognitively impaired within \pm 12 months of PA assessment ( $n=323)$; 3 . had no record of one or more items of participant characteristics (age, sex, race, etc.) mentioned above ( $\mathrm{n}=85)$; 4 . had no follow-up SIS assessment after the date of wearing the accelerometer $(n=338) ; 5$. had a stroke before the most recent follow-up SIS assessment $(\mathrm{n}=202)$. A final sample of 6,452 participants with usable accelerometer data and at least one complete follow-up SIS measure were included in the analyses. 


\section{Measures}

An Actical ${ }^{\mathrm{TM}}$ (Mini Mitter Respironics, Inc. Bend, OR) worn over the right hip while attached to a neoprene waistband provided estimates of the frequency, intensity, and duration of PA. Participants were instructed to put on the device after waking up each morning and take it off prior to going to bed each evening. Participants were instructed to wear the device for 7 consecutive days and return the device immediately after. For all participants with usable data, absolute time spent in sedentary behavior (SED), light intensity PA (LPA) and moderate-to-vigorous intensity PA (MVPA), and proportions of total accelerometer wear time spent in SED (SED\%), LPA (LPA\%), and MVPA (MVPA\%) were determined. Nonwear periods were defined as a string of zero counts per minute $(\mathrm{cpm})$ for $>120$ consecutive minutes (21). Activity count cut-points were applied to differentiate SED (0-49 cpm), LPA (50-1064 cpm), and MVPA (>1065 cpm), respectively $(17,21,37)$. A lab-based validation study for Actical ${ }^{\mathrm{TM}}$ was completed among older adults with similar demographic characteristics to our study (17).

Cognitive function was assessed during REGARDS follow-up telephone interviews using standardized scripts and scoring methods described previously (33). Cognitive impairment was defined by each participant's score on the SIS. Four expanded cognitive battery tests with demonstrated usefulness in early identification of cognitive decline, Alzheimer's disease or dementia were also utilized (26). These tests were Word List Learning (WLL) and semantic fluency (animal fluency, AF) from the Consortium to Establish a Registry for Alzheimer's Disease battery (26), and letter fluency (LF), recall and orientation items from the Montreal Cognitive Assessment (MoCA-recall and orientation) (27). These tools assess domains of memory (WLL, MoCA-recall and orientation) and executive function (AF, LF) $(12,26)$. Z-scores based on the mean and standard deviation for each specific test in domains of memory and executive function were created, respectively. The average z-score of both tests under each domain were used as the z-score of memory or executive function. Participants were included if they had at least one baseline measure in memory or executive function domain within \pm 12 months of PA assessment, and at least one follow-up measure of the same test after the PA assessment (37). All cognitive assessments have been proven to be valid and reliable for both white and black participants $(12,26,27,33)$ with no significant racial/ethnic differences having been reported.

\section{Statistical Analysis}

Continuous data expressed as MVPA (min/wk) and MVPA\% were skewed, thereby introducing concerns regarding the highly leveraged points. Thus, the primary independent variable of MVPA\% was divided into quartiles allowing for group comparisons and investigation of the dose-response relationship. Due to inherent variability in daily accelerometer wear time, MVPA\% rather than the absolute time spent in MVPA was used as the standard for quartiles to render results comparable among individuals. The main dependent variable was incident cognitive impairment defined as a shift from intact cognitive screening status (SIS score of 5 or 6 ) at the closest assessment to the baseline PA measurement (within \pm 12 months) to impaired cognitive screening status (SIS score of $\leq 4$ ) at the latest follow-up assessment. Secondary dependent variables were the changes in z- 
scores of memory and executive function. Analyses for this report were based on data acquired through April, 2015.

Differences in demographic variables, accelerometer variables, and cognitive tests across quartiles of MVPA\% were tested by analysis of variance or Chi-square tests. Logistic regression analysis was used to estimate odds ratios (ORs) of cognitive impairment and the associated $95 \%$ CIs, with adjustments made for age, sex, race, region of residence, education, BMI, hypertension, smoking, and diabetes. General linear regression models with adjustment for confounders mentioned above, baseline scores, and follow-up time intervals were used to assess the association between PA and changes in z-scores generated from WLL, AF, LF and MoCA-recall and orientation. Results were also stratified by race in this analysis. Regression analysis including the interaction terms (i.e., sex*PA, race*PA) was also conducted, and there were no significant interactions between MVPA\% and these confounders, respectively $(\mathrm{P}>0.05)$. Thus, interaction terms were not included in the final models. All probability values were based on 2-tailed tests; $\mathrm{P}<0.05$ indicated statistical significance. Analyses were conducted using SAS version 9.4 (SAS Institute, Cary, NC).

\section{Results}

Demographics, accelerometer data, and cognitive function status are displayed in Tables 1 and 2 according to quartiles of MVPA\%. Among the 6,452 participants, 55.3\% were women, and $30.5 \%$ were black. The mean $( \pm$ SD) age was $69.7 \pm 8.5$ years. Participants were compliant wearing the accelerometer for $6.6 \pm 0.8$ days. Participants spent most of their wear time in SED $(77.1 \pm 9.3 \%)$ and LPA $(21.4 \% \pm 8.4 \%)$. MVPA $\%$ was extremely limited $(1.5 \% \pm 1.9 \%)$. Participants with higher MVPA $\%$ were significantly more likely to be men, white, younger, well-educated, non-smoker, without diabetes or hypertension, and lower in BMI and blood pressure. Participants with higher MVPA\% had significantly better raw scores in WLL, AF, LF, and MoCA-recall and orientation, and higher baseline z-scores in executive function and memory $(\mathrm{P}<0.001)$.

During a mean $( \pm \mathrm{SD})$ follow-up period of $2.9 \pm 1.1$ years, 346 incident cases of cognitive impairment occurred according to SIS. White adults experienced 209 and black adults 137 cases of incident cognitive impairment, respectively. Higher MVPA\% was significantly associated with less decreases in the cognitive scores of WLL, AF, LF, MoCA-recall and orientation, executive function and memory z-scores during follow-up (Table 3). The association between MVPA\% and risk of cognitive impairment was significant in white and black adults combined (Table 4). These relationships remained significant after adjustment for age, sex, race, region of residence, and education in Model 2 (Quartile 2: OR=0.63, 95\% $\mathrm{CI}=0.48-0.84$; Quartile 3: $\mathrm{OR}=0.53,95 \% \mathrm{CI}=0.38-0.74$; Quartile 4: $\mathrm{OR}=0.57,95 \%$ $\mathrm{CI}=0.40-0.81$ ). In Model 3, results were consistent with Model 2, with participants in the higher MVPA\% quartiles less likely to develop cognitive impairment. Stratified analyses indicated higher MVPA\% quartiles were independently associated with lower risk of cognitive impairment among white adults in all models. However, there was no significant association between MVPA\% and risk of cognitive impairment in black adults in the adjusted models (i.e. Quartile 4: $\mathrm{OR}=0.69,95 \% \mathrm{CI}=0.36-1.32$ in Model 3). Sensitivity analyses were conducted with a minimum of two years between baseline assessment of PA 
and the next available SIS to exclude any underlying diseases at baseline, but most of the results were identical. Similar analyses were conducted using LPA\% and SED\% quartiles, but no significant results were found for any of the cohort groupings (see eTable 1 , Supplemental Digital Content 1, association of LPA\% and SED\% with incident cognitive impairment).

Adjusted linear regression models revealed some significant associations between MVPA\% and changes in z-scores for executive function and memory when controlling for age, sex, race, region of residence, education, BMI, hypertension, smoking, diabetes, baseline scores, and follow-up time intervals (Table 5). Compared with the lowest MVPA\% quartiles, all other quartiles maintained better memory performance $(\mathrm{P}<0.05)$, while only the highest quartile significantly differed in maintenance of executive function $(\mathrm{P}=0.026)$. In race stratified analyses, higher MVPA\% quartiles were independently associated with better maintenance of executive function (Quartile 4 of MVPA\%) and memory (Quartile 2, 3 and 4 of MVPA\%) $(\mathrm{P}<0.05)$ in white adults. In black adults, higher MVPA\% quartiles were independently associated with better maintenance of memory (Quartile 3 and 4 of MVPA\%; $\mathrm{P}<0.05)$, but not executive function $(\mathrm{P}>0.05)$. Preservation of memory over time was of similar magnitude among black and white adults. Similar analyses were conducted using LPA\% and SED\% quartiles, but no significant results were observed (see eTable 2, Supplemental Digital Content 2, association of LPA\% with change in cognitive z-scores; and eTable 3, Supplemental Digital Content 3, association of SED\% with change in cognitive zscores).

\section{Discussion}

This study is one of the first to examine the dose-response association between objectively measured PA and cognitive function in a large and racially/ethnically diverse older population. Our study is unique in demonstrating a significant relationship between objectively measured PA and cognitive function among 6,452 white and black older adults during follow-up. Even a minor difference in MVPA\% (i.e., only a 3-5 min mean difference between Quartile 1 and Quartile 2) was associated with a significantly $36 \%$ lower risk of incident cognitive impairment and greater maintenance of executive function and memory over time. The study also indicated higher MVPA\% was independently associated with a $39-47 \%$ less risk of cognitive impairment and maintenance of memory and executive function over time in white adults, as well as maintenance of memory performance in black adults. These findings confirm our previous cross-sectional analysis in the same cohort (37), and support a dose-response relationship exists between engagement in MVPA\% and cognitive performance over time. Interventions should be provided to prevent cognitive decline before symptoms are manifest.

The association between PA and cognitive function has been studied previously $(11,34)$. A meta-analysis (13) of 16 prospective studies using self-reported PA indicated the relative risks of incident dementia and Alzheimer's disease in the higher PA categories were significantly reduced by $28-45 \%$. However, these previous studies were limited by the potential bias of self-report measures of PA, and also have not been able to explore the effects of LPA and SED on cognitive function. A few studies with objectively measured data 
have reported active older adults may have lower risks of cognitive impairment and dementia. One cross-sectional study (15) showed total accelerometer measured PA (step counts per week) was positively associated with visual episodic memory and face-name memory accuracy in older adults. Another (8) reported greater MVPA (hr/day) was related to lower volume of white matter lesions. In a recent longitudinal study, Barnes et al. (3) demonstrated higher levels of aerobic fitness $\left(\mathrm{VO}_{2 \max }\right)$ predicted better performance on measures of attention and executive function during 6 years of follow-up. Middleton et al. (25) reported a significant dose-response relationship between activity energy expenditure measured by doubly labeled water and incidence of cognitive impairment over 2-5 years of follow-up. In the Rush Memory and Aging Project, accelerometer measured total daily PA (counts/day) was substantially negatively associated with cognitive decline and incident Alzheimer's Disease in older individuals without dementia during a follow-up period of 4 years (6). The sample sizes of these longitudinal studies were relatively small $(\mathrm{N}=197-716)$ and primarily focused on white Americans. Additionally, the methodological differences in objective measures preclude comparisons of studies of the optimal pattern of PA to prevent cognitive decline. With this current analysis, we can more confidently suggest even a small dose of daily free-living MVPA, rather than self-reported PA or structured exercise in clinical settings, was significantly associated with maintenance of cognitive performance.

In the current study, analyses revealed the association between PA and cognitive function remained robust in older adults after multiple adjustments, which confirms previous findings $(24,35)$. The Chicago Health and Aging Project reported more self-report PA was modestly (not independently) associated with less cognitive decline in a large community of white and black older adults over 6 years (30). In our REGARDS cohort, the stratified analyses indicated a similar association of MVPA\% with memory performance over time among white and black adults, but with executive function among only white adults. To our knowledge, this is one of the first studies to investigate how race/ethnicity may moderate the relationship between objectively measured PA and cognitive function in older adults. Further observation of this cohort over time is needed to better determine if disparities exist between white and black adults with respect to the PA and cognitive function dose-response relationship across specific cognitive domains.

Interestingly, the results regarding LPA\% and SED\% revealed no significant relationships with cognitive function change over time. These findings suggest PA intensity may also be an important independent factor influencing the relationship between PA and cognitive function in older adults. Other studies applying accelerometer measurements have reported intensity of PA is influential in the association between PA and memory and executive function in older adults $(5,22)$. Previous studies have reported a significant association between LPA and various health measures (7), indicating additional follow-up may be necessary to investigate the association LPA and cognitive function. For SED, computer using time has been positively associated with memory and executive function, while television viewing time was negatively associated with cognitive function in older adults $(14,16,23)$. Unfortunately, we were not able to determine the nature of sedentary behavior in our study. Due to conflicting results in this emerging area, additional investigations are necessary to fully explore the impact of SED on cognitive change. 
This study has several strengths. First, objectively measured PA was used to examine the association of PA with cognitive function in a large population of white and black older adults. This allowed for analyses with proportion of time spent being sedentary and in PA of varying intensity. Second, the sample was recruited from a well-characterized cohort of midlife and older black and white adults living in the U.S., and a substantial percentage of participants were quite compliant. Third, we explored the relationships of accelerometer derived PA with specific domains of memory and executive function, in addition to odds of impairment in global cognition. Fourth, this is one of the first studies to stratify results on the relationship between objectively measured PA and cognitive function by race.

The findings of this study were subject to limitations. Disadvantages of using hip-worn accelerometers include not being able to identify types of PA or capture upper-body movement. The application of standard cut-points to differentiate time spent being sedentary and physically active at varying intensities does not account for potential differences in fitness levels, especially among older adults. Thus, the measured time in MVPA in our study may be underestimated. Also, the cohort was quite sedentary (>11 hr/day; $77 \%$ of accelerometer wearing time) and there was a significant skewness of MVPA\%. Limited variability of the dependent variable and trend of the association makes it difficult to determine a precise threshold of MVPA for reducing risk of cognitive impairment. There were no substantial differences by race-sex groups in those agreeing to participate in the accelerometer protocol compared with those who did not, with the exception that those who participated had higher education level (20). In addition, there were differences by race in the final proportions of usable data with blacks providing a smaller yield (20). Thus, the results may not be generalizable to other REGARDS participants.

\section{Conclusion}

Higher levels of objectively measured MVPA\% were independently associated with lower incidence of cognitive impairment in white older adults, with the greatest reduction in risk between the lowest and next highest MVPA\% quartiles (i.e., as little as $4 \mathrm{~min} /$ day or 28 $\mathrm{min} / \mathrm{wk}$ of MVPA may be helpful in maintaining cognitive function). Higher levels of MVPA\% were also independently and incrementally associated with better maintenance of memory over time for both black and white adults, as well as maintenance of executive function for white adults. Race may moderate the relationship between PA and certain aspects of cognitive function in older adults. Neither LPA\% nor SED\% were independently associated with any measures of cognitive function in this cohort.

\section{Supplementary Material}

Refer to Web version on PubMed Central for supplementary material.

\section{Acknowledgments}

This work is supported by a cooperative agreement U01 NS041588 and investigator- initiated grant R01NS061846 from the National Institute of Neurological Disorders and Stroke, National Institutes of Health, Department of Health and Human Service. Additional funding was supported by the Fundamental Research Funds for the Central Universities (GK201603128) and an unrestricted research grant from The Coca-Cola Company. The sponsors had no role in the design and conduct of the study; collection, management, analysis, and interpretation of the data; 
preparation, review, or approval of the manuscript; and decision to submit the manuscript for publication. The authors thank the other investigators, the staff, and the participants of the REGARDS study for their valuable contributions. A full list of participating REGARDS investigators and institutions can be found at http:// www.regardsstudy.org. We also acknowledge the contributions of Dr Barbara Ainsworth, Dr Matthew Buman, and Dr Cheryl der Ananian who served on the first author's doctoral dissertation committee at Arizona State University.

\section{References}

1. Angevaren M, Vanhees L, Nooyens AC, Wendel-Vos CG, Verschuren WM. Physical activity and 5year cognitive decline in the Doetinchem cohort study. Ann Epidemiol. 2010; 20(6):473-9. [PubMed: 20470975]

2. Baker LD, Frank LL, Foster-Schubert K, et al. Aerobic exercise improves cognition for older adults with glucose intolerance, a risk factor for Alzheimer's disease. J Alzheimers Dis. 2010; 22(2):569_ 79. [PubMed: 20847403]

3. Barnes DE, Yaffe K, Satariano WA, Tager IB. A longitudinal study of cardiorespiratory fitness and cognitive function in healthy older adults. J Am Geriatr Soc. 2003; 51(4):459-65. [PubMed: 12657064]

4. Bopp M, Wilcox S, Laken M, et al. Factors associated with physical activity among AfricanAmerican men and women. Am J Prev Med. 2006; 30(4):340-6. [PubMed: 16530622]

5. Brown BM, Peiffer JJ, Sohrabi HR, et al. Intense physical activity is associated with cognitive performance in the elderly. Transl Psychiatry. 2012; 2:e191. [PubMed: 23168991]

6. Buchman AS, Boyle PA, Yu L, Shah RC, Wilson RS, Bennett DA. Total daily physical activity and the risk of AD and cognitive decline in older adults. Neurology. 2012; 78(17):1323-9. [PubMed: 22517108]

7. Buman MP, Hekler EB, Haskell WL, et al. Objective light-intensity physical activity associations with rated health in older adults. Am J Epidemiol. 2010; 172(10):1155-65. [PubMed: 20843864]

8. Burzynska AZ, Chaddock-Heyman L, Voss MW, et al. Physical activity and cardiorespiratory fitness are beneficial for white matter in low-fit older adults. PLoS One. 2014; 9(9):e107413. [PubMed: 25229455]

9. Callahan CM, Unverzagt FW, Hui SL, Perkins AJ, Hendrie HC. Six-item screener to identify cognitive impairment among potential subjects for clinical research. Med Care. 2002; 40(9):771-81. [PubMed: 12218768]

10. Davis MG, Fox KR. Physical activity patterns assessed by accelerometry in older people. Eur J Appl Physiol. 2007; 100(5):581-9. [PubMed: 17063361]

11. Geda YE, Roberts RO, Knopman DS, et al. Physical exercise and mild cognitive impairment: A population-based study. Arch Neurol. 2010; 67(1):80-6. [PubMed: 20065133]

12. Hachinski V, Iadecola C, Petersen RC, et al. National Institute of Neurological Disorders and Stroke-Canadian Stroke Network vascular cognitive impairment harmonization standards. Stroke. 2006; 37(9):2220-41. [PubMed: 16917086]

13. Hamer M, Chida Y. Physical activity and risk of neurodegenerative disease: A systematic review of prospective evidence. Psychol Med. 2009; 39(1):3-11. [PubMed: 18570697]

14. Hamer M, Stamatakis E. Screen-based sedentary behavior, physical activity, and muscle strength in the English longitudinal study of ageing. PLoS One. 2013; 8(6):e66222. [PubMed: 23755302]

15. Hayes SM, Alosco ML, Hayes JP, et al. Physical activity is positively associated with episodic memory in aging. J Int Neuropsychol Soc. 2015; 21(10):780-90. [PubMed: 26581790]

16. Hoang TD, Reis J, Zhu N, et al. Effect of early adult patterns of physical activity and television viewing on midlife cognitive function. JAMA Psychiatry. 2016; 73(1):73-9. [PubMed: 26629780]

17. Hooker SP, Feeney A, Hutto B, et al. Validation of the Actical activity monitor in middle-aged and older adults. J Phys Act Health. 2011; 8(3):372-81. [PubMed: 21487136]

18. Hooker SP, Hutto B, Zhu W, et al. Accelerometer measured sedentary behavior and physical activity in white and black adults: The REGARDS study. J Sci Med Sport. 2015; 19(4):336-41. [PubMed: 25937313] 
19. Howard VJ, Cushman M, Pulley L, et al. The reasons for geographic and racial differences in stroke study: Objectives and design. Neuroepidemiology. 2005; 25(3):135-43. [PubMed: 15990444]

20. Howard VJ, Rhodes JD, Mosher A, et al. Obtaining accelerometer data in a national cohort of black and white adults. Med Sci Sports Exerc. 2015; 47(7):1531-7. [PubMed: 25333247]

21. Hutto B, Howard VJ, Blair SN, et al. Identifying accelerometer nonwear and wear time in older adults. Int J Behav Nutr Phys Act. 2013; 10:120. [PubMed: 24156309]

22. Kerr J, Marshall SJ, Patterson RE, et al. Objectively measured physical activity is related to cognitive function in older adults. J Am Geriatr Soc. 2013; 61(11):1927-31. [PubMed: 24219194]

23. Kesse-Guyot E, Charreire H, Andreeva VA, et al. Cross-sectional and longitudinal associations of different sedentary behaviors with cognitive performance in older adults. PLoS One. 2012; 7(10):e47831. [PubMed: 23082222]

24. Landi F, Russo A, Barillaro C, et al. Physical activity and risk of cognitive impairment among older persons living in the community. Aging Clin Exp Res. 2007; 19(5):410-6. [PubMed: 18007121]

25. Middleton LE, Manini TM, Simonsick EM, et al. Activity energy expenditure and incident cognitive impairment in older adults. Arch Intern Med. 2011; 171(14):1251-7. [PubMed: 21771893]

26. Morris JC, Heyman A, Mohs RC, et al. The consortium to establish a registry for Alzheimer's disease (CERAD). Part I. clinical and neuropsychological assessment of Alzheimer's disease. Neurology. 1989; 39(9):1159-65. [PubMed: 2771064]

27. Nasreddine ZS, Phillips NA, Bedirian V, et al. The Montreal cognitive assessment, MoCA: A brief screening tool for mild cognitive impairment. J Am Geriatr Soc. 2005; 53(4):695-9. [PubMed: 15817019]

28. Nelson ME, Rejeski WJ, Blair SN, et al. Physical activity and public health in older adults: Recommendation from the American College of Sports Medicine and the American Heart Association. Med Sci Sports Exerc. 2007; 39(8):1435-45. [PubMed: 17762378]

29. Sofi F, Valecchi D, Bacci D, et al. Physical activity and risk of cognitive decline: A meta-analysis of prospective studies. J Intern Med. 2011; 269(1):107-17. [PubMed: 20831630]

30. Sturman MT, Morris MC, Mendes de Leon CF, Bienias JL, Wilson RS, Evans DA. Physical activity, cognitive activity, and cognitive decline in a biracial community population. Arch Neurol. 2005; 62(11):1750-4. [PubMed: 16286550]

31. Tierney MC, Moineddin R, Morra A, Manson J, Blake J. Intensity of recreational physical activity throughout life and later life cognitive functioning in women. J Alzheimers Dis. 2010; 22(4):13318. [PubMed: 20930291]

32. Troiano RP. Physical activity in the United States measured by accelerometer. Med Sci Sports Exerc. 2008; 40(1):181-8. [PubMed: 18091006]

33. Wadley VG, Unverzagt FW, McGuire LC, et al. Incident cognitive impairment is elevated in the stroke belt: The REGARDS study. Ann Neurol. 2011; 70(2):229-36. [PubMed: 21618586]

34. Willey JZ, Gardener H, Caunca MR, et al. Leisure-time physical activity associates with cognitive decline: The Northern Manhattan Study. Neurology. 2016; 86(20):1897-903. [PubMed: 27009261]

35. Winker R, Lukas I, Perkmann T, et al. Cognitive function in elderly marathon runners: Crosssectional data from the marathon trial (APSOEM). Wien Klin Wochenschr. 2010; 122(23-24):70416. [PubMed: 21072603]

36. Wortmann M. Dementia: A global health priority - highlights from an ADI and World Health Organization Report. Alzheimers Res Ther. 2012; 4(5):40. [PubMed: 22995353]

37. Zhu W, Howard VJ, Wadley VG, et al. Association between objectively measured physical activity and cognitive function in older adults-the reasons for geographic and racial differences in stroke study. J Am Geriatr Soc. 2015; 63(12):2447-54. [PubMed: 26691697] 


\section{D.}

ชิ

.

胥

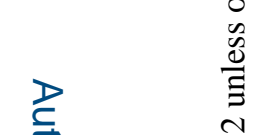

을

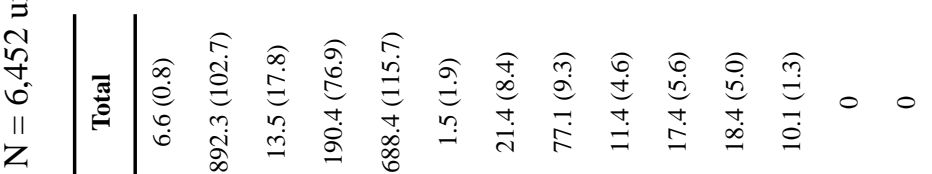

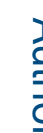

要

西

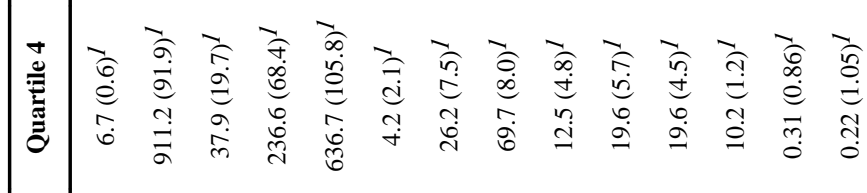

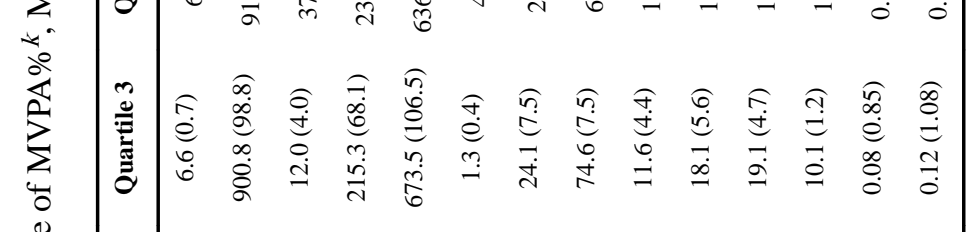

索

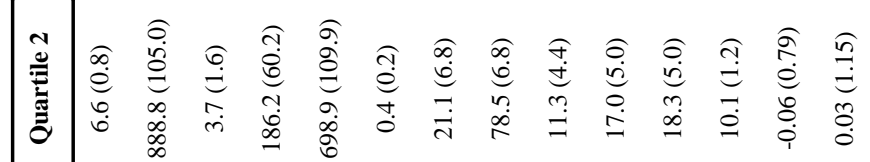

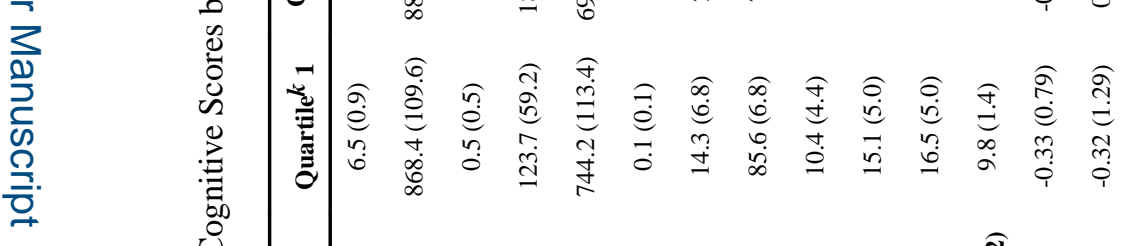

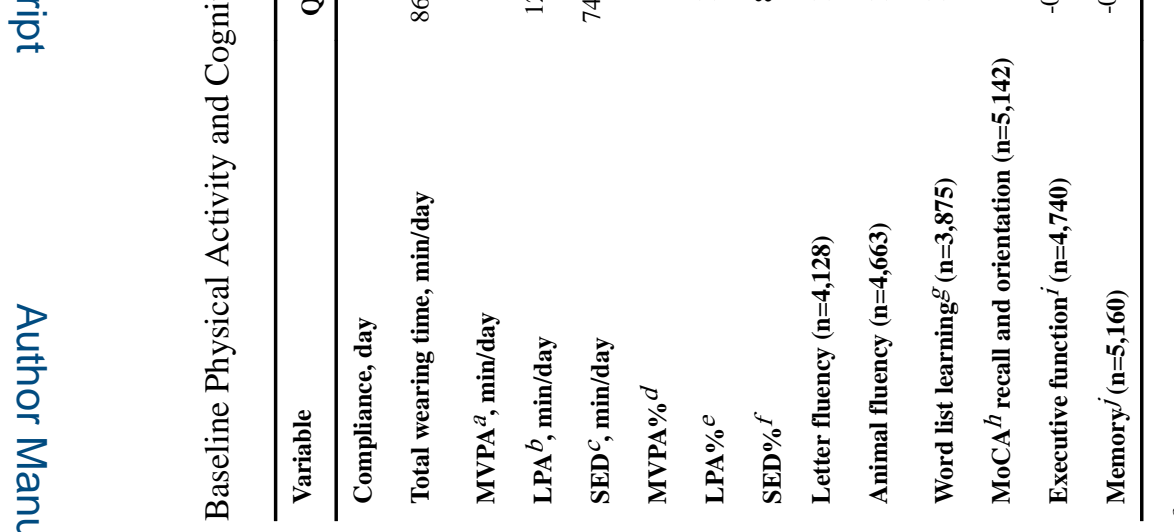

옴

Med Sci Sports Exerc. Author manuscript; available in PMC 2018 January 01. 


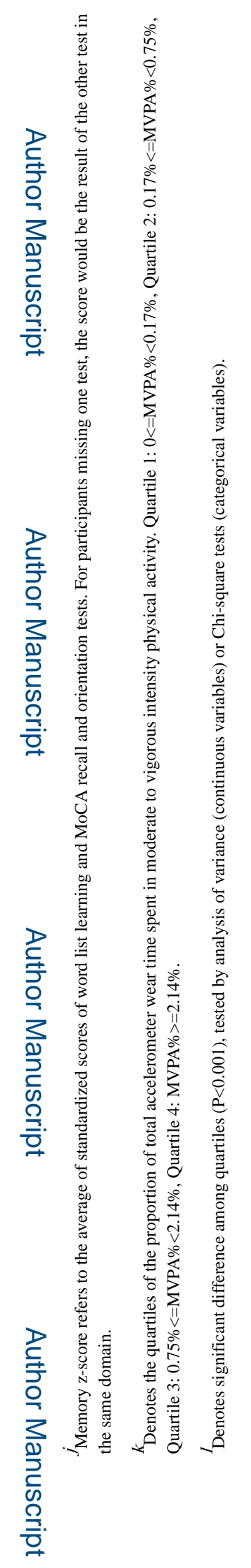

Med Sci Sports Exerc. Author manuscript; available in PMC 2018 January 01. 
Table 4

Incident Cognitive Impairment by Quartiles of MVPA\% ${ }^{f}(\mathrm{~N}=6,452)$ in Follow-up

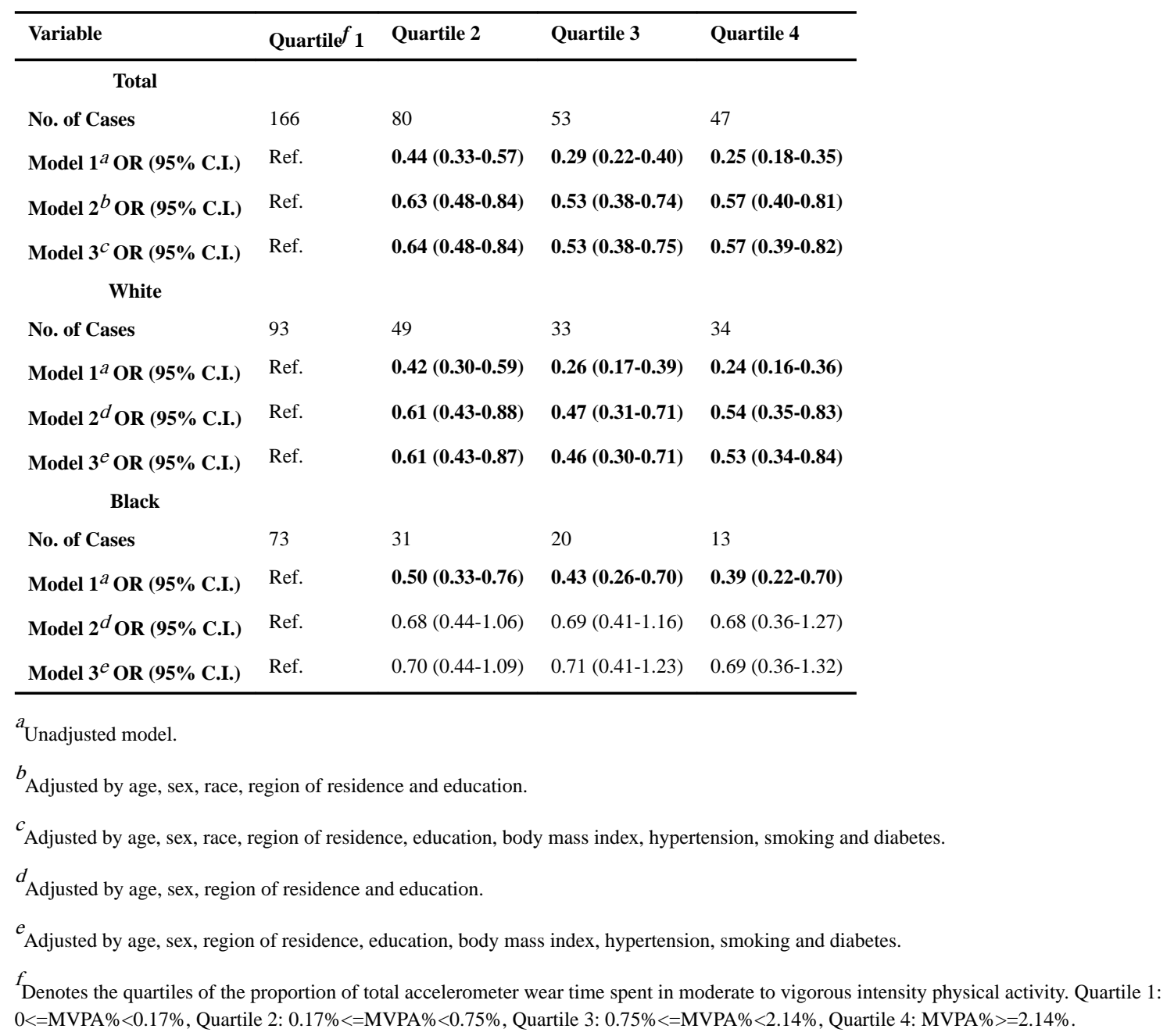




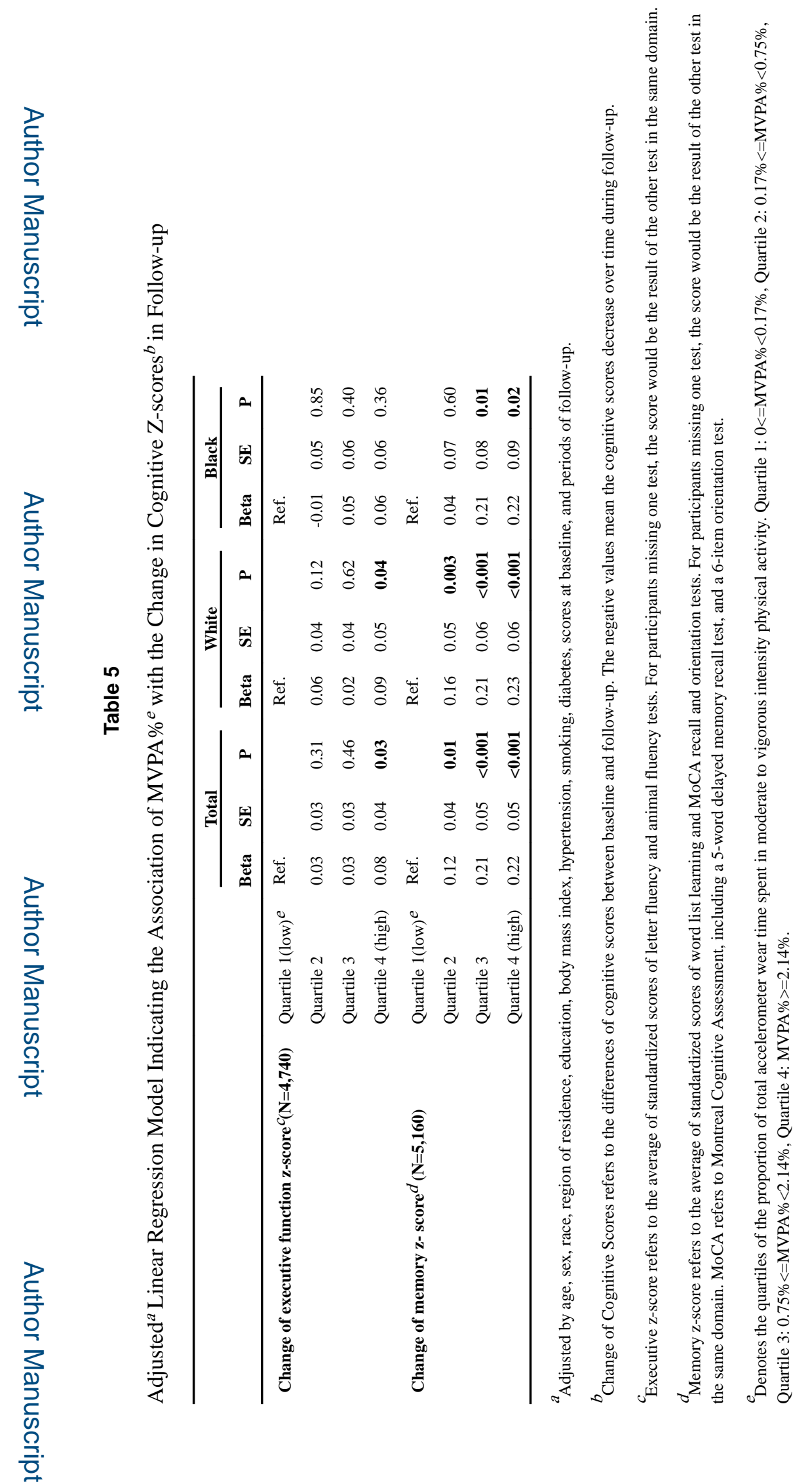

Med Sci Sports Exerc. Author manuscript; available in PMC 2018 January 01. 